\title{
Disorders of the Optic Nerve in Mitochondrial Cytopathies: New Ideas on Pathogenesis and Therapeutic Targets
}

\author{
Kamil S. Sitarz • Patrick F. Chinnery • \\ Patrick Yu-Wai-Man
}

Published online: 4 March 2012

(C) The Author(s) 2012. This article is published with open access at Springerlink.com

\begin{abstract}
Mitochondrial cytopathies are a heterogeneous group of human disorders triggered by disturbed mitochondrial function. This can be due to primary mitochondrial DNA mutations or nuclear defects affecting key components of the mitochondrial machinery. Optic neuropathy is a frequent disease manifestation and the degree of visual failure can be profound, with a severe impact on the patient's quality of life. This review focuses on the major mitochondrial disorders exhibiting optic nerve involvement, either as the defining clinical feature or as an additional component of a more extensive phenotype. Over the past decade, significant progress has been achieved in our basic understanding of Leber hereditary optic neuropathy and autosomaldominant optic atrophy-the two classical paradigms for these mitochondrial optic neuropathies. There are currently limited treatments for these blinding ocular disorders and, ultimately, the aim is to translate these major advances into tangible benefits for patients and their families.
\end{abstract}

Keywords Dominant optic atrophy · Haplogroup . Hereditary spastic paraplegia, Heteroplasmy - Idebenone . Leber hereditary optic neuropathy · Mitochondrial DNA .

K. S. Sitarz $\cdot$ P. F. Chinnery $\cdot$ P. Yu-Wai-Man

Wellcome Trust Centre for Mitochondrial Research, Institute of Genetic Medicine, Newcastle University, Newcastle, UK

P. Yu-Wai-Man

Department of Ophthalmology, Royal Victoria Infirmary,

Newcastle upon Tyne, UK

P. Yu-Wai-Man $(\bowtie)$

Wellcome Trust Centre for Mitochondrial Research, Institute of Genetic Medicine, Centre for Life, Newcastle University, Newcastle upon Tyne NE1 3BZ, UK

e-mail: Patrick.Yu-Wai-Man@ncl.ac.uk
Mitofusin · Multiple sclerosis · Neuroprotection · Retinal ganglion cell $\cdot$ Optic nerve $\cdot$ Mitochondrial cytopathies

\section{Introduction}

Mitochondria are found in all nucleated cells and reflecting this ubiquitous presence, patients with mitochondrial cytopathies often manifest a diverse combination of tissue and organ involvement [1]. However, for reasons that still remain unclear, mitochondrial dysfunction has a marked predilection for the optic nerve, the latter being affected in about half of all patients with confirmed mitochondrial disease (Fig. 1). Irrespective of the molecular pathways involved, remarkably, these mitochondrial optic neuropathies all share the same pathological features-selective degeneration of the retinal ganglion cell (RGC) layer, leading to progressive axonal loss and the onset of visual failure [2]. In this review, recent advances in our understanding of this important group of disorders are discussed, in addition to promising therapeutic strategies.

\section{Leber Hereditary Optic Neuropathy}

Epidemiology

Leber hereditary optic neuropathy (LHON, OMIM 535000) is named after Theodore Leber (1840-1917), a German ophthalmologist who was the first to describe the key features of this disorder [3]. LHON is the most common of the primary mitochondrial DNA (mtDNA) disorders and the minimum prevalence has been estimated at 1 in 31,000 in the North of England (Fig. 1) [4]. Epidemiological studies from the Netherlands and Finland have reported comparable 
Figure 1 Mitochondrial disease in the North of England. (Adapted from Yu-Wai-Man et al. [2] and Schaefer et al. [50])

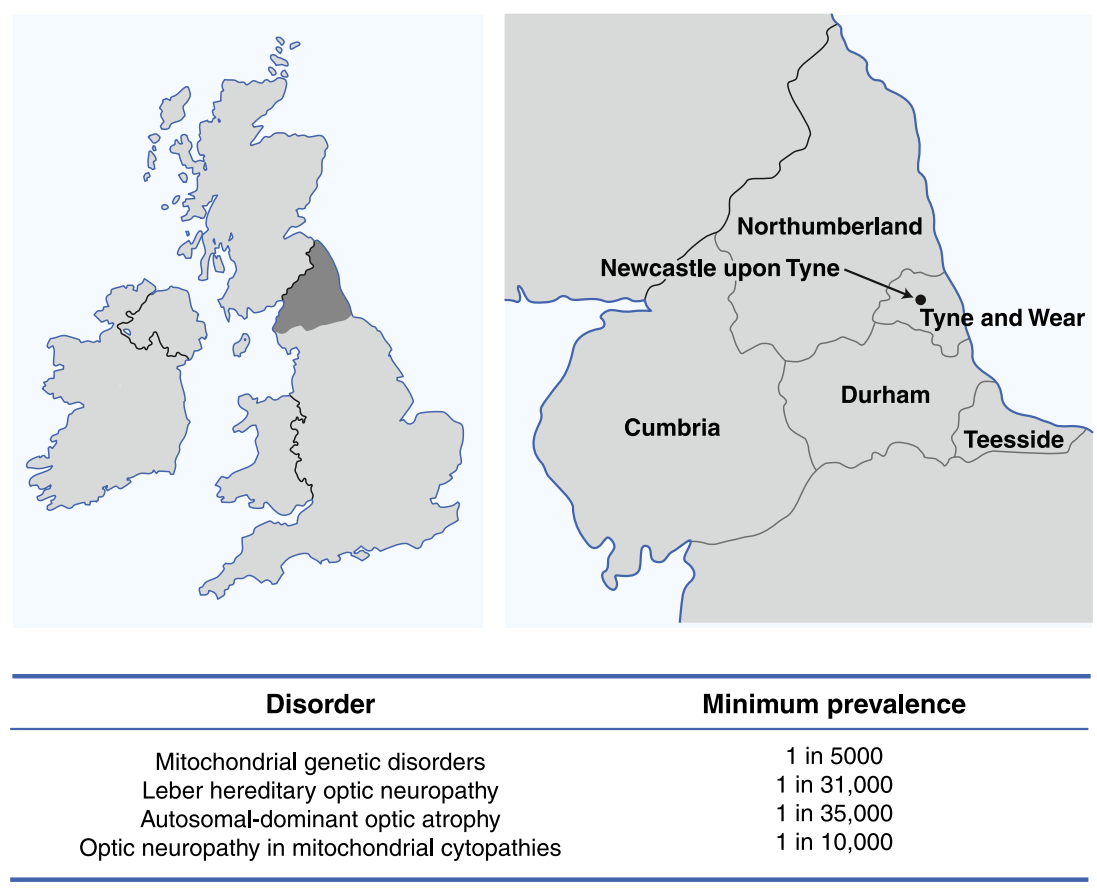

figures of 1 in 39,000 and 1 in 50,000, respectively. In one Australian study, $2 \%$ of individuals on the National Blind Registry had optic atrophy secondary to LHON, highlighting the significant socioeconomic burden of this inherited optic nerve disorder. Three mtDNA point mutations $-\mathrm{m} .3460 \mathrm{G}>$ A, m.11778 G>A, and m.14484 T $>\mathrm{C}$ - account for the vast majority ( $\sim 90 \%$ ) of LHON cases and, for this reason, they are often referred to as "primary" (Table 1) [3, 5].

\section{Clinical Manifestations}

Typically, disease conversion in LHON is characterized by acute or subacute central visual loss in one eye, followed 2 to 4 months later by the fellow eye $[3,5]$. Unilateral optic nerve involvement in LHON is exceptionally rare and another underlying pathological process should be actively excluded in these atypical cases. Bilateral simultaneous onset probably occurs in about $25 \%$ of patients, although it can be difficult for some individuals to accurately report whether visual loss had been ongoing in one eye prior to the fellow eye being affected. The peak age of onset is in the second and third decades of life and it is unusual for symptoms to develop beyond 50 years of age.

The initial visual loss in LHON is severe and it usually plateaus over the next 6 months, with most patients achieving visual acuities of $20 / 200$ or worse $[3,5]$. There is an associated dense central or centrocecal scotoma and color vision is significantly impaired. The pupillary light reflexes are relatively preserved and this rather surprising feature has recently been ascribed to a special class of melanopsincontaining RGCs that are more resistant to the mtDNA
LHON mutations $[6 \bullet \bullet]$. In the acute phase, classically, there is optic disc hyperemia, peripapillary telangiectatic vessels, vascular tortuosity, and retinal nerve fiber layer (RNFL) edema secondary to axonal stasis. In a proportion $(20 \%$ to $40 \%$ ) of LHON carriers undergoing disease conversion, the optic discs can look entirely normal, followed 6 weeks later by the development of neuroretinal rim pallor [5]. Pathological cupping of the optic disc is also a recognized feature of longstanding LHON cases, reflecting the ongoing loss of RGC axons in the chronic phase of the disease. The overall prognosis in LHON is poor, even among patients harboring the m.14484 $\mathrm{T}>\mathrm{C}$ mutation, which carries the best chance for partial visual recovery [5]. If it occurs, a slow improvement in visual parameters can be expected within the first year, although more delayed visual recovery has been reported.

\section{Syndromal LHON Phenotypes}

LHON is typically a monosymptomatic disease but additional features such as cardiac conduction defects, peripheral neuropathy, dystonia, and myopathy have been reported as occurring more frequently among LHON carriers. In particular, there is a well-reported association between the three primary mtDNA LHON mutations and a multiple sclerosislike illness, especially among female carriers. Rarer pathogenic mtDNA variants have been linked with more atypical "LHON +" syndromes, where the optic neuropathy segregated with prominent neurological features including spastic dystonia, ataxia, juvenile-onset encephalopathy, and psychiatric disturbances [2]. 
Table 1 mtDNA variants associated with LHON

\begin{tabular}{|c|c|c|}
\hline & $\begin{array}{l}\text { Mitochondrial } \\
\text { gene }\end{array}$ & Nucleotide change \\
\hline \multirow{3}{*}{$\begin{array}{l}\text { Common variants } \\
\quad(\sim 90 \%)\end{array}$} & MTND1 & $\mathrm{m} .3460 \mathrm{G}>\mathrm{A}^{\mathrm{a}}$ \\
\hline & MTND4 & $\mathrm{m} .11778 \mathrm{G}>\mathrm{A}^{\mathrm{a}}$ \\
\hline & MTND6 & $\mathrm{m} .14484 \mathrm{~T}>\mathrm{C}^{\mathrm{a}}$ \\
\hline \multirow[t]{10}{*}{$\begin{array}{l}\text { Rare variants } \\
\qquad(\sim 10 \%)\end{array}$} & MTND1 & $\begin{array}{r}\mathrm{m} .3376 \mathrm{G}>\mathrm{A}, \mathrm{m} .3635 \mathrm{G}>\mathrm{A}^{\mathrm{a}} \\
\mathrm{m} .3697 \mathrm{G}>\mathrm{A}, \mathrm{m} .3700 \mathrm{G}>\mathrm{A} \\
\mathrm{m} .3733 \mathrm{G}>\mathrm{A}^{\mathrm{a}}, \mathrm{m} .4025 \mathrm{C}>\mathrm{T} \\
\mathrm{m} .4160 \mathrm{~T}>\mathrm{C}, \mathrm{m} .4171 \mathrm{C}>\mathrm{A}^{\mathrm{a}}\end{array}$ \\
\hline & MTND2 & $\mathrm{m} .4640 \mathrm{C}>\mathrm{A}, \mathrm{m} .5244 \mathrm{G}>\mathrm{A}$ \\
\hline & MTND3 & m.10237 T>C \\
\hline & MTND4 & m.11696 G>A, m.11253 T>C \\
\hline & MTND4L & $\mathrm{m} .10663 \mathrm{~T}>\mathrm{C}^{\mathrm{a}}$ \\
\hline & MTND5 & $\begin{array}{l}\mathrm{m} .12811 \mathrm{~T}>\mathrm{C}, \mathrm{m} .12848 \mathrm{C}>\mathrm{T}, \\
\mathrm{m} .13637 \mathrm{~A}>\mathrm{G}, \mathrm{m} .13730 \mathrm{G}>\mathrm{A}\end{array}$ \\
\hline & MTND6 & $\begin{array}{l}\mathrm{m} .14325 \mathrm{~T}>\mathrm{C}, \mathrm{m} .14568 \mathrm{C}>\mathrm{T}, \\
\mathrm{m} .14459 \mathrm{G}>\mathrm{A}^{\mathrm{a}}, \mathrm{m} .14729 \mathrm{G}>\mathrm{A}, \\
\mathrm{m} .14482 \mathrm{C}>\mathrm{A}^{\mathrm{a}}, \mathrm{m} .14482 \mathrm{C}> \\
\mathrm{G}^{\mathrm{a}}, \\
\mathrm{m} .14495 \mathrm{~A}>\mathrm{G}^{\mathrm{a}}, \mathrm{m} .14498 \mathrm{C}>\mathrm{T}, \\
\mathrm{m} .14568 \mathrm{C}>\mathrm{T}^{\mathrm{a}}, \mathrm{m} .14596 \mathrm{~A}>\mathrm{T}\end{array}$ \\
\hline & MTATP6 & $\mathrm{m} .9101 \mathrm{~T}>\mathrm{C}$ \\
\hline & MTCO3 & m.9804 G>A \\
\hline & $M T C Y B$ & m.14831 G>A \\
\hline
\end{tabular}

Over $70 \%$ of LHON carriers harbor the $\mathrm{m} .11778 \mathrm{G}>\mathrm{A}$ mutation, but as a result of a founder event, the m.14484 $\mathrm{T}>\mathrm{C}$ mutation has been identified in nearly $90 \%$ of all affected patients of French Canadian descent $[3,5]$. In most laboratories worldwide, the diagnostic protocol involves screening for the three "primary" LHON mutations in the first instance. As full mitochondrial genome sequencing remains time consuming and expensive, this is only indicated if the initial LHON screen is negative and there is a strong clinical suspicion

${ }^{a}$ These mtDNA variants are definitely pathogenic. They have been identified in two or more independent LHON pedigrees, showing segregation with affected disease status. The remaining putative LHON mutations have been found in singleton cases or in a single family, and additional evidence is required before pathogenicity can be irrefutably ascribed

LHON Leber hereditary optic neuropathy; $m t D N A$ mitochondrial DNA

\section{Disease Modifiers}

LHON is characterized by incomplete penetrance and a marked sex bias, with only about $50 \%$ of male carriers and about $10 \%$ of female carriers losing vision during their lifetime. The pathological consequences of the mtDNA LHON mutations are clearly being influenced by other disease modifiers, and these can be classified into four main groups: 1) the mitochondrial genetic background, 2) nuclear susceptibility genes, 3) hormonal differences, and 4) environmental triggers.

As mitochondria have a high copy number genome, LHON carriers can be homoplasmic (100\% mutant) for the mtDNA mutation, or heteroplasmic with a combination of both the wild-type and mutant mtDNA species. Although the risk of visual loss is minimal at heteroplasmy levels below $60 \%$, this observation cannot account for the bulk of unaffected LHON carriers, most of whom are homoplasmic mutant and therefore beyond this biochemical threshold [7]. MtDNA is highly polymorphic and during human evolution, ancient mtDNA variants have clustered together in specific combinations known as haplogroups. As mtDNA haplogroups influence mitochondrial oxidative phosphorylation, these more subtle variations could magnify or lessen the impact of the LHON mutation on RGC survival. In a large study involving 159 Caucasian LHON pedigrees, haplogroup J was associated with a significantly increased risk of visual loss among m.11778 $\mathrm{G}>\mathrm{A}$ and $\mathrm{m} .14484 \mathrm{~T}>\mathrm{C}$ carriers, whereas m.3460 G>A carriers were more likely to become affected on a haplogroup $\mathrm{K}$ background [8]. Haplogroup $\mathrm{H}$ had a protective effect but only among individuals harboring the m.11778 $\mathrm{G}>\mathrm{A}$ mutation. Haplogroup associations have been reported in other ethnic groups, supporting a contributory role for the mtDNA background in influencing LHON penetrance [9].

The predilection for males to lose vision in LHON cannot be explained by mitochondrial genetics and a visual loss susceptibility gene on the $\mathrm{X}$ chromosome has long been suspected, the so-called two-locus disease model [10]. Although such an X-linked disease modifier gene has yet to be formally identified, three independent linkage studies have revealed overlapping candidate regions, providing strong evidence for its existence [11-13]. Another obvious factor to account for the observed male bias in LHON is a protective influence conferred by female sex hormones. This hypothesis was recently investigated using LHON cybrid cell lines, and interestingly, treatment with $17 \beta$-estradiol resulted in reduced reactive oxygen species (ROS) levels, increased activity of the antioxidant enzyme superoxide dismutase, and more efficient mitochondrial biogenesis [14•].

Various environmental factors have been implicated in precipitating visual loss among LHON carriers including head trauma, industrial toxins, and drugs with mitochondrial toxic effects [15]. The evidence for these environmental triggers is largely anecdotal, but a recent multicenter study of 125 LHON pedigrees has provided convincing evidence for an increased risk of visual failure among smokers, and to a lesser extent, heavy drinkers [16•].

\section{Mitochondrial Encephalomyopathies}

The mitochondrial encephalomyopathies encompass several distinct phenotypes such as mitochondrial encephalomyopathy, lactic acidosis, and stroke-like episodes (MELAS), myoclonic epilepsy and ragged-red fibers (MERRF), maternally inherited Leigh syndrome (MILS), mitochondrial neurogastrointestinal encephalomyopathy (MNGIE), and the Kearns-Sayre syndrome (KSS) [1, 
17]. Although variable and not a disease-defining feature, the occurrence of optic atrophy is well described in this group of patients, exacerbating the already considerable disease burden [18]. Additional studies are required to determine the true prevalence of both clinical and subclinical optic neuropathy in these mitochondrial encephalomyopathies, and whether specific mtDNA mutations or phenotypes are linked with an increased risk of optic nerve involvement.

\section{Nuclear Mitochondrial Disorders}

Autosomal-Dominant Optic Atrophy

\section{Epidemiology}

Frederick Batten (1865-1918), a pediatric neurologist, is credited with the first report of autosomal-dominant optic atrophy (DOA) in one British family. The characteristic features were described in greater detail by Poul Kjer in a large cohort of Danish families, establishing DOA as a distinct clinical entity from LHON [3]. The prevalence of DOA was estimated at 1 in 35,000 in the North of England (Fig. 1), but additional families have since been identified and the true figure is likely to be much higher (Yu-Wai-Man, Unpublished data).

\section{Disease Genes and Candidate Loci}

Between $50 \%$ and $60 \%$ of families with DOA harbor pathogenic mutations in $O P A 1$, which codes for a mitochondrial inner membrane protein (Table 2) [3,5]. OPA1 is highly polymorphic and over 200 pathogenic variants have been identified, clustering in the GTPase region and the dynamic central domain. To minimize cost, OPA1 is routinely sequenced using polymerase chain reaction-based methods, which will not detect exonic deletions or duplications. These large-scale rearrangements have been identified in $10 \%$ to
$20 \%$ of $O P A 1$-negative families, and these additional molecular studies, although not yet widely available, are recommended for probands with a clear-cut DOA phenotype and a strong family history [2].

Mutations in OPA3 were first described in Iraqi-Jewish families with autosomal-recessive type III 3-methylglutaconic aciduria (Costeff syndrome), a progressive neurodegenerative disorder with early-onset optic atrophy, hypotonia, ataxia, extrapyramidal dysfunction, and cognitive decline [19]. Heterozygous $O P A 3$ mutations were subsequently reported in two French families with a dominantly inherited form of optic atrophy associated with premature cataract formation (ADOAC) [20]. Similar to OPA1, OPA3 has a mitochondrialtargeting domain and it was initially considered to be a mitochondrial inner membrane protein. However, a recent study has suggested instead that OPA3 localizes to the mitochondrial outer membrane, with its C-terminus facing the cytosol [21]. The causative genetic defects in the remaining families with DOA have not yet been identified, although a number of candidate loci have been reported (Table 2).

\section{Visual Function and Disease Progression}

Visual loss in DOA is bilateral and symmetrical with the majority patients reporting subnormal vision from early childhood. There is a wide intra- and inter-familial variability in disease severity, with visual acuities ranging from 20/20 to the detection of hand movement $[2,3]$. Individuals with visual acuities of 20/30 or better are frequently asymptomatic and they are only identified as having features of a bilateral optic neuropathy at the time of contact tracing. On long-term follow-up, visual function was observed to deteriorate in $50 \%$ to $75 \%$ of patients with DOA but the rate of progression was highly variable, making genetic counseling difficult $[2$, 3]. Although a milder optic nerve disease compared with LHON, DOA still results in significant visual impairment with nearly half of all patients eventually registered blind.

As in LHON, the primary site of pathology in DOA is the papillomacular bundle and central, centrocecal, and

Table 2 Nuclear mitochondrial disorders with prominent optic nerve involvement

\begin{tabular}{lllll}
\hline Inheritance & Locus & Gene & OMIM & Phenotypes \\
\hline Dominant & $1 \mathrm{p} 36.2$ & MFN2 & 601152 & Hereditary motor and sensory neuropathy type 6 (HMSN-6, CMT2A) \\
& $3 \mathrm{q} 28-\mathrm{q} 29$ & OPA1 & 165500 & Isolated optic atrophy and syndromal dominant optic atrophy (DOA+) \\
& $19 \mathrm{q} 13.2-\mathrm{q} 13.3$ & OPA3 & 165300 & Autosomal-dominant optic atrophy and early-onset cataracts (ADOAC) \\
Recessive & $9 \mathrm{q} 13-\mathrm{q} 21.1$ & $F X N$ & 229300 & Friedreich's ataxia (FRDA) \\
& $11 \mathrm{q} 14.1-\mathrm{q} 21$ & TMEM126A & 612989 & Optic atrophy \pm auditory neuropathy \\
& $16 \mathrm{q} 24.3$ & SPG7 & 607259 & Hereditary spastic paraplegia type 7 (HSP-7) \\
& $19 \mathrm{q} 13.2-\mathrm{q} 13.3$ & OPA3 & 258501 & Type III 3-methylglutaconic aciduria (Costeff syndrome) \\
\hline
\end{tabular}

(Adapted from Yu-Wai-Man et al. [2, 5]) 
paracentral scotomas are the most common visual field defects. The degree of dyschromatopsia is commensurate with the level of vision and pure tritanopia, once considered a pathognomonic feature of DOA, is only rarely seen. Histopathological studies of a postmortem eye retrieved from one patient showed relative preservation of melanopsincontaining RGCs, accounting for the lack of an afferent pupillary defect in DOA - another peculiarity shared with LHON [6••]. The optic nerve head in DOA can look diffusely pale or it can have a characteristic temporal wedge, especially in patients with early disease where RGC loss remains mostly localized to the papillomacular bundle $[2,3]$.

\section{The Expanding Phenotypic Spectrum}

Up to $20 \%$ of patients harboring $O P A 1$ mutations will develop a syndromal form of the disease (DOA+) marked by significant neurological complications [22•]. The most common extraocular feature observed in this group is sensorineural hearing loss, which usually manifests itself in the second and third decades of life after visual failure has become apparent. A proportion of OPAl carriers will progress to a more debilitated state with variable combinations of ataxia, peripheral neuropathy, myopathy, and progressive external ophthalmoplegia in later life. From a mechanistic perspective, it is also revealing that some patients with DOA + can present with clinical features indistinguishable from other neurodegenerative disorders such as multiple sclerosis, hereditary spastic paraplegia (HSP), and the inherited spinocerebellar degenerations. The phenotypic spectrum of OPA1 disease is likely to expand even further with greater clinical awareness and easier access to molecular genetic testing.

\section{Other Nuclear Mitochondrial Optic Neuropathies}

The nuclear defects underlying several common neuromuscular disorders have been clarified. A remarkable element has been the increasing realization that mitochondrial dysfunction plays a central role in the pathophysiology of disease groups as diverse as Charcot-Marie-Tooth (CMT) disease, HSP, and the inherited spinocerebellar ataxiasreflecting to a certain extent the broader phenotypic spectrum recently described for $O P A 1$ disease [2]. Mutations in $M F N 2$, which codes for a critical mitochondrial outer membrane protein, have been identified in patients with hereditary motor and sensory neuropathy type 6 (HMSN-6), an autosomal-dominant axonal CMT subtype (Table 2) [23]. Affected individuals develop an early-onset peripheral neuropathy with a progressive bilateral optic atrophy in later childhood. Optic neuropathy also features prominently in Friedreich ataxia and HSP type 7 (HSP-7), caused by nuclear mutations in $F X N$ and $S P G 7$, respectively [2]. Frataxin is essential for the assembly of the iron-sulfur clusters embedded within the mitochondrial respiratory chain complexes, whereas paraplegin is a key mitochondrial protease involved in the proteolytic processing of OPA1 [24, 25]. All these disease pathways are likely interrelated, and disturbance in one element will set into motion a vicious cycle, which eventually disturbs mitochondrial homeostasis triggering neuronal cell loss. As the molecular basis of other inherited human diseases is uncovered, it will be interesting to note whether optic atrophy is present in those cases where the genetic defect is ultimately revealed to affect mitochondrial function. A recent example is the identification of TMEM126A mutations among patients with autosomalrecessive optic atrophy, with or without an associated auditory neuropathy [26]. Although its exact functions still remain to be determined, fascinatingly, TMEM126A is a mitochondrial transmembrane protein present at high levels within the RGC layer and the optic nerve head [26].

\section{Mechanisms Contributing to RGC Loss}

The final pathological outcome in mitochondrial optic neuropathies is apoptotic RGC loss and several disease pathways can contribute to this irreversible process. Although a greater emphasis has been placed on the experimental data obtained for LHON and DOA, similar mechanisms have also been shown to operate in other mitochondrial cytopathies.

\section{Bioenergetic Failure}

Mitochondria are the cell's powerhouses providing most of its adenosine triphosphate (ATP) requirements through the tight control of mitochondrial respiratory chain activity. All three primary LHON mutations - $\mathrm{m} .3460 \mathrm{G}>\mathrm{A}, \mathrm{m} .11778 \mathrm{G}>\mathrm{A}$, and $\mathrm{m} .14484 \mathrm{~T}>\mathrm{C}$ - disrupt key polypeptide subunits of complex I. Similarly, OPA1 mutations have a detrimental impact on mitochondrial oxidative output by impairing the assembly and stability of the respiratory chain complexes [27]. Most studies, based on in vitro or in vivo assays, indicate a significant impairment in complex I activity, leading to a reduction in mitochondrial membrane potential and overall ATP synthesis (reviewed in [5] and [15]). Although this bioenergetic deficit is bound to impact negatively on RGCs, by itself, this cannot account for their selective vulnerability. Photoreceptors, for example, have a much higher energetic demand and outer retinal function is usually preserved in both LHON and DOA.

\section{ROS and Excitotoxicity}

The disrupted flow of high-energy electrons along the respiratory chain leads to free radical production and increased 
ROS levels have been consistently observed in transmitochondrial LHON cybrids (reviewed in [5] and [15]). The efficient reuptake of glutamate is also disrupted in these cellular models due to the downregulation of excitatory amino acid transporter (EAAT1) activity. A drosophila Opal (dOpal) model has recently been established and homozygous mutant flies developed a rough and glossy eye phenotype due to the loss of hexagonal lattice cells, with decreased lens and pigment deposition. This genetically engineered $d O p a l$ mutation led to a dramatic increase in ROS levels, which could be partially rescued with dietary antioxidant supplementation or overexpression of SOD1 [28]. Heterozygous adult flies were phenotypically normal but similar to the homozygotes, ROS levels were elevated with clear evidence of cellular oxidative stress. Increased ROS levels and glutamate excitotoxicity clearly represent a toxic combination, sufficiently potent to initiate the apoptotic cascade.

\section{Disturbed Calcium Handling}

Besides its localization to the mitochondrial outer membrane, MFN2 is also associated with the endoplasmic reticulum (ER), resulting in a close communication between the mitochondrial and ER membranes [29••, 30]. When the MFN2 protein is disrupted or absent, as in CMT-2A this tethering effect is lost with dramatic consequences on cellular calcium homeostasis. The ER and mitochondrial network are important calcium stores and the dynamic flux between these two compartments buffers against cytosolic calcium spikes, which can sensitize the cell to various proapoptotic signals such as glutamate [29••, 30]. Mirroring the pathomechanism of MFN2 mutations, mtDNA point mutations involving complex I and complex IV subunits have also been directly linked with disturbed calcium handling in neuronal populations [31]. Although speculative, it is likely that a similar process is operating in LHON and DOA, contributing to RGC dysfunction and ultimately disease conversion among at-risk mutational carriers [32]. The emerging links between calcium homeostasis and mitochondrial dysfunction are an exciting new development, highlighting potential disease pathways amenable to therapeutic intervention.

\section{Mitochondrial Network Dynamics}

Cytochrome $c$ is a powerful pro-apoptotic mediator and as a protective mechanism, high concentrations of these molecules are carefully sequestered within the mitochondrial cristae. Irrespective of the mtDNA mutation involved, the dissipation of the mitochondrial membrane potential accelerates the cytosolic release of these cytochrome $c$ molecules, precipitating an irreversible commitment toward programmed cell death [33]. The identification of OPA1 and MFN2 mutations in DOA and CMT-2A has provided further insights into this key disease mechanism. OPA1 ensures the integrity of the mitochondrial cristae's tight junctions, preventing cytochrome $c$ from leaching out into the cytosolic space. In addition, OPA1 and MFN2 both belong to the dynamin superfamily of mechanoenzymes, sharing considerable structural similarities, including a highly conserved catalytic GTPase domain [24]. These two proteins have important pro-fusional properties and by working closely together, they maintain a highlyinterconnected mitochondrial network throughout the cell's structure [24, 33]. Unsurprisingly, the pathological hallmark of OPA1- and MFN2-mutant fibroblasts is mitochondrial network fragmentation, which not only impairs mitochondrial oxidative phosphorylation, but also leads to the uncontrolled release of calcium and cytochrome $c$ into an already compromised cellular environment $[27,34,35 \bullet \cdot]$.

\section{Somatic mtDNA Defects}

Pathological levels of cytochrome $c$ oxidase-negative fibers have been identified in skeletal muscle biopsies from patients with DOA+ $[35 \bullet \bullet, 36 \bullet \cdot$. The underlying biochemical defect in these muscle fibers is secondary to the accumulation of high levels of somatic mtDNA deletions, which have clonally expanded during the patient's lifetime, partly accounting for the delayed onset of these extraocular features $[37 \bullet \bullet]$. Intriguingly, the risk of developing DOA + is three times higher with missense mutations targeting the functional GTPase domain, consistent with a dominantnegative mutational effect [22•]. OPA1 is clearly involved in preserving the integrity of the mitochondrial genome and recent studies have provided some tantalizing insights into the mechanisms that contribute to mtDNA instability [38], $[39 \bullet \bullet, 40 \bullet \cdot$. OPA1 is thought to anchor the mtDNA replicative machinery, known as nucleoids, to the mitochondrial inner membrane and a dominant-negative mutation could plausibly upset this delicate balance, leading to mtDNA deletion formation $[40 \bullet \cdot$. Why is this relevant to our understanding of RGC loss? Patients with DOA+ have worse visual acuities and significantly thinner RNFL thickness compared with those who only develop isolated optic atrophy [41]. The accumulation of these somatic mtDNA deletions is therefore clearly having an incremental effect on the other deleterious consequences linked to OPA1 mutations.

\section{Why Are RGCs Selectively Vulnerable?}

One factor that could explain this tissue-specific vulnerability is the rather unusual anatomical peculiarity encountered at the lamina cribrosa, where RGC axons first acquire their 
myelin sheaths. This transition is marked by a sharp differential gradient, with a much higher density of mitochondria and voltage-gated sodium channels in the pre-laminar unmyelinated segment of the optic nerve [42, 43]. These physiological adaptations facilitate the efficient propagation of action potentials in the absence of an insulating myelin covering. The pre-laminar region therefore represents a weak link, significantly more exposed to the disadvantageous consequences of, even subtle, mitochondrial biochemical defects.

Histopathological studies of optic nerves retrieved from two patients with LHON, one harboring the m.3460 G>A mutation and the other the m.11778 $\mathrm{G}>\mathrm{A}$ mutation, have revealed some interesting observations on the susceptibility of specific RGC populations (reviewed in [2] and [15]). A prominent loss of smaller-caliber axons was observed, corresponding to the parvocellular RGC population, whereas the larger-caliber magnocellular RGCs were relatively preserved. These ultrastructural findings were subsequently confirmed with higher-resolution transmission electron microscopy. Parvocellular RGCs are a major component of the papillomacular bundle and this greater vulnerability to impaired mitochondrial oxidative phosphorylation could be related to their relatively smaller cross-sectional areas, the latter further exacerbating axonal stasis in conditions of sustained metabolic stress.

The maintenance of a higher mitochondrial concentration in the pre-laminar region also highlights the central role played by the cytoskeleton, especially the microtubule network, in channeling mitochondria to their appropriate cellular locations. Axonal transport in highly specialized neuronal populations such as RGCs is critically dependent on these mitochondrial-cytoskeletal interactions (reviewed in [2] and [15]). These can be adversely affected either by an underlying mitochondrial respiratory chain defect, or by a primary disturbance in microtubule assembly - the pathological hallmark of the HSP group of disorders [2]. Optic atrophy is well described in HSP-7 and it is likely that optic nerve involvement remains an under-reported feature in other HSP genetic subtypes.

\section{Therapeutic Interventions}

\section{Visual Rehabilitation}

The majority of patients with mitochondrial optic neuropathies are severely visually impaired and the sudden onset of visual loss in otherwise healthy individuals carries a significant psychological and socioeconomic burden. Therefore, clinicians have an important role to play in facilitating access to rehabilitative services such as low visual aids and occupational therapy.
Clinical Surveillance

It is essential to screen for associated systemic complications, such as diabetes and cardiomyopathy, among patients with mitochondrial cytopathies. The development of diabetic peripheral neuropathy and cardiac-related exertional dyspnea can further compound the physical difficulties faced by patients with poor vision, and they should be aggressively managed as part of a multidisciplinary team. As a general health measure, patients with mitochondrial disease should be advised not to smoke and to moderate their alcohol intake. Furthermore, in LHON, smoking, and to a lesser extent excessive binge drinking, have been linked with an increased risk of visual loss [16•].

\section{Pharmacological Agents}

Various pharmacological agents with putative neuroprotective properties have been used to mitigate the deleterious consequences of mitochondrial dysfunction and to prevent further RGC loss (reviewed in [44••]). The most promising agent to date is idebenone (2,3-dimethoxy-5-methyl-6-[10hydroxydecyl]-1,4-benzoquinone), a short-chain synthetic benzoquinone analogue, which promotes mitochondrial ATP synthesis in addition to having antioxidant properties. Coenzyme $\mathrm{Q} 10\left(\mathrm{CoQ}_{10}\right)$ is a longer-chain quinone analogue and based on limited evidence, $\mathrm{CoQ}_{10}$ supplementation is often used to treat patients with mitochondrial cytopathies. Idebenone is thought to have some advantageous properties over $\mathrm{CoQ}_{10}$, both in terms of its bioavailability and its mode of action. Unlike $\mathrm{CoQ}_{10}$, idebenone is able to bypass complex I inhibition by shuttling electrons directly from the cytosol to complex III, thereby restoring ATP production and decreasing lactate levels [45].

The results of a multicenter randomized placebocontrolled trial (RHODOS [Rescue of Hereditary Optic Disease Outpatient Study]) of idebenone in LHON have recently been released. Idebenone was well tolerated at a dose of $900 \mathrm{mg}$ per day and no adverse drug-related events were reported. The visual outcome data indicate that patients with discordant visual acuities ( $\log M A R>0.2)$, and thus at highest risk of further deterioration in the least affected eye, were more likely to benefit from treatment with idebenone [44••]. The findings from the RHODOS trial are promising and for the first time, they offer the hope of preserving vision for affected LHON carriers who are still at an early stage of the disease process.

\section{Looking into the Future: Gene Therapy}

Gene therapy for primary mitochondrial cytopathies poses several discrete challenges; the mitochondrial inner membrane is a relatively impermeable membrane that needs to be 
bypassed, and a highly efficient vector is needed to transfect a sufficient number of mitochondria per cell to achieve the desired effect. A possible solution is to bypass the mitochondrial genome using an allotopic approach. Instead, the gene of interest is transfected into the nuclear genome and the protein product is engineered with a specific targeting sequence that facilitates its uptake into the mitochondrial compartment, thereby compensating for the mtDNA mutation. Proof of principle has been demonstrated in experimental LHON models where adeno-associated virus vectors have been used to successfully transfect cells with a replacement wild-type mtDNA allele or neuroprotective genes such as SOD2 [46-48]. These results are encouraging and allotopic rescue could be easily applied to the RGC layer which is easily accessible. However, key issues of safety and efficacy need to be further addressed before their application to human clinical trials can be advocated [2].

\section{Preventing Disease Transmission}

Several strategies are currently being explored to prevent the transmission of pathogenic mitochondrial and nuclear mutations among women of child-bearing age. The difficult technical and ethical issues raised by preimplantation genetic testing and new in vitro fertilization techniques such as pronuclear transfer are outside the scope of this review, and these have been detailed elsewhere $[2,49]$.

\section{Conclusions}

A number of recurring disease mechanisms have been identified that contribute to RGC loss in mitochondrial optic neuropathies. These provide a unique opportunity for targeted therapeutic interventions aimed not only at improving visual function, but also the neurological deficits seen in the more severe mitochondrial cytopathies. However, despite these major advances, the risk factors underpinning the selective vulnerability of RGCs have yet to be clarified. Research in this area has been severely limited by the lack of diseased human optic nerves but hopefully, with the development of faithful animal models and more advanced biotechnological tools, we will soon be in a position to disentangle these fundamental research questions both at the structural and molecular levels.

Acknowledgments P. Yu-Wai-Man is a Medical Research Council (MRC, UK) Clinician Scientist in Neuro-Ophthalmology and P.F. Chinnery is a Wellcome Trust Senior Fellow in Clinical Science. P.F. Chinnery also receives funding from Parkinson's UK, the MRC Translational Muscle Centre, and the UK NIHR Biomedical Research Centre in Ageing and Age-related Disease.
Disclosure No potential conflicts of interest relevant to this article were reported.

Open Access This article is distributed under the terms of the Creative Commons Attribution License which permits any use, distribution, and reproduction in any medium, provided the original author(s) and the source are credited.

\section{References}

Papers of particular interest, published recently, have been highlighted as:

- Of importance

•- Of major importance

1. McFarland R, Taylor RW, Turnbull DM. The neurology of mitochondrial DNA disease. Lancet Neurol. 2002;1(6):343-51.

2. Yu-Wai-Man P, Griffiths PG, Chinnery PF. Mitochondrial optic neuropathies-Disease mechanisms and therapeutic strategies. Progress in Retinal and Eye Research. 2011;30(2):81114.

3. Fraser JA, Biousse V, Newman NJ. The Neuro-ophthalmology of Mitochondrial Disease. Surv Ophthalmol. 2010;55(4):299-334.

4. Man PY, Griffiths PG, Brown DT, et al. The epidemiology of Leber hereditary optic neuropathy in the North East of England. Am J Hum Genet. 2003;72(2):333-9.

5. Yu-Wai-Man P, Griffiths PG, Hudson G, Chinnery PF. Inherited mitochondrial optic neuropathies. Journal of Medical Genetics. 2009;46(3):145-58.

6. • La Morgia C, Ross-Cisneros FN, Sadun AA, et al.: Melanopsin retinal ganglion cells are resistant to neurodegeneration in mitochondrial optic neuropathies. Brain. 2010;133:2426-38. This paper describes the preservation of a special class of melanopsincontaining RGCs in LHON and DOA, providing for the first time a neuroanatomical basis for the relatively spared pupillary light reflexes in these two disorders.

7. Chinnery PF, Andrews RM, Turnbull DM, Howell NN. Leber hereditary optic neuropathy: Does heteroplasmy influence the inheritance and expression of the G11778A mitochondrial DNA mutation? American Journal of Medical Genetics. 2001;98 (3):235-43.

8. Hudson G, Carelli V, Spruijt L, et al. Clinical expression of Leber hereditary optic neuropathy is affected by the mitochondrial DNAhaplogroup background. Am J Hum Genet. 2007;81(2):228-33.

9. Ji YL, Zhang AM, Jia XY, et al. Mitochondrial DNA Haplogroups M7b1 ' 2 and M8a Affect Clinical Expression of Leber Hereditary Optic Neuropathy in Chinese Families with the m.11778 G $\rightarrow$ A Mutation. Am J Hum Genet. 2008;83(6):760-8.

10. $\mathrm{Bu} X \mathrm{XD}$, Rotter JI: X chromosome-linked and mitochondrial gene control of Leber hereditary optic neuropathy: evidence from segregation analysis for dependence on $\mathrm{X}$ chromosome inactivation. Proceedings of the National Academy of Sciences of the United States of America. 1991;88(18):8198-202.

11. Hudson G, Keers S, Man PYW, et al. Identification of an Xchromosomal locus and haplotype modulating the phenotype of a mitochondrial DNA disorder. Am J Hum Genet. 2005;77(6):108691.

12. Shankar SP, Fingert JH, Carelli V, et al. Evidence for a novel xlinked modifier locus for leber hereditary optic neuropathy. Ophthalmic Genetics. 2008;29(1):17-24. 
13. Ji YL, Jia XY, Li SQ, et al. Evaluation of the X-linked modifier loci for Leber hereditary optic neuropathy with the G11778A mutation in Chinese. Mol Vis. 2010;16(47):416-24.

14. - Giordano C, Montopoli M, Perli E, et al.: Oestrogens ameliorate mitochondrial dysfunction in Leber's hereditary optic neuropathy. Brain. 2011;134:220-34. This paper describes an elegant series of experiments showing that estrogens help protect LHON cybrids against the deleterious consequences of the three primary mtDNA mutations. These results support a hormonal component to the reduced disease penetrance observed among female LHON carriers.

15. Carelli V, Ross-Cisneros FN, Sadun AA. Mitochondrial dysfunction as a cause of optic neuropathies. Progress in Retinal and Eye Research. 2004;23(1):53-89.

16. - Kirkman MA, Yu-Wai-Man P, Korsten A, et al.: Geneenvironment interactions in Leber hereditary optic neuropathy. Brain. 2009;132:2317-26. This is the largest epidemiological study to investigate the role of environmental factors in influencing LHON penetrance. A strong association was identified between the risk of visual loss and smoking. This effect showed a dose-response relationship with heavy smokers being more likely to be affected than light smokers. A trend toward increased visual failure was also noted among LHON carriers with heavy alcohol consumption.

17. Schapira AHV. Mitochondrial disease. Lancet. 2006;368 (9529):70-82.

18. Gronlund MA, Honarvar AKS, Andersson S, et al. Ophthalmological findings in children and young adults with genetically verified mitochondrial disease. $\mathrm{Br} \mathrm{J}$ Ophthalmol. 2010;94(1):121-7.

19. Anikster Y, Kleta R, Shaag A, et al. Type III 3methylglutaconic aciduria (optic atrophy plus syndrome, or Costeff optic atrophy syndrome): Identification of the OPA3 gene and its founder mutation in Iraqi Jews. Am J Hum Genet. 2001;69(6):1218-24.

20. Reynier P, Amati-Bonneau P, Verny C, et al. OPA3 gene mutations responsible for autosomal dominant optic atrophy and cataract. Journal of Medical Genetics. 2004;41(9):e110.

21. Ryu SW, Jeong HJ, Choi M, et al. Optic atrophy 3 as a protein of the mitochondrial outer membrane induces mitochondrial fragmentation. Cellular and Molecular Life Sciences. 2010;67 (16):2839-50.

22. • Yu-Wai-Man P, Griffiths PG, Gorman GS, et al.: Multi-system neurological disease is common in patients with OPA1 mutations. Brain. 2010;133:771-86. This large multicenter study expands the phenotypic spectrum associated with OPA1 mutations. Up to $20 \%$ of mutational carriers developed a more severe disease variant (DOA+) characterized by prominent neuromuscular features such as deafness, myopathy, peripheral neuropathy, ataxia, and chronic progressive external ophthalmoplegia. Interestingly, there was a threefold increased risk of developing DOA+ with missense OPA1 mutations involving the GTPase domain compared with other mutational subgroups.

23. Zuchner S, De Jonghe P, Jordanova A, et al. Axonal neuropathy with optic atrophy is caused by mutations in mitofusin 2 . Ann Neurol. 2006;59(2):276-81.

24. Lenaers G, Reynier P, Elachouri G, et al. OPA1 functions in mitochondria and dysfunctions in optic nerve. Int J Biochem Cell Biol. 2009;41(10):1866-74.

25. Stemmler TL, Lesuisse E, Pain D, Dancis A. Frataxin and Mitochondrial FeS Cluster Biogenesis. J Biol Chem. 2010;285 (35):26737-43.

26. Hanein S, Perrault I, Roche O, et al. TMEM126A, Encoding a Mitochondrial Protein, Is Mutated in Autosomal-Recessive Nonsyndromic Optic Atrophy. Am J Hum Genet. 2009;84 (4):493-8.
27. Zanna C, Ghelli A, Porcelli AM, et al. OPA1 mutations associated with dominant optic atrophy impair oxidative phosphorylation and mitochondrial fusion. Brain. 2008;131:352-67.

28. Tang S, Le PK, Tse S, et al. Heterozygous Mutation of Opal in Drosophila Shortens Lifespan Mediated through Increased Reactive Oxygen Species Production. PLoS One. 2009;4(2): e4492.

29. •- de Brito OM, Scorrano L: Mitofusin 2 tethers endoplasmic reticulum to mitochondria. Nature. 2008;456(7222):605-10. This seminal work describes how MFN2 tethers the ER to the mitochondrial network, thereby regulating calcium flux between these two compartments.

30. de Brito OM, Scorrano L. Mitofusin-2 regulates mitochondrial and endoplasmic reticulum morphology and tethering: The role of Ras. Mitochondrion. 2009;9(3):222-6.

31. Trevelyan AJ, Kirby DM, Smulders-Srinivasan TK, et al. Mitochondrial DNA mutations affect calcium handling in differentiated neurons. Brain. 2010;133:787-96.

32. Dayanithi G, Chen-Kuo-Chang M, Viero C, et al. Characterization of $\mathrm{Ca} 2+$ signalling in postnatal mouse retinal ganglion cells: involvement of OPA1 in Ca2+ clearance. Ophthalmic Genet. 2010;31(2):53-65.

33. Westermann B. Mitochondrial fusion and fission in cell life and death. Nat Rev Mol Cell Biol. 2010;11(12):872-84.

34. Chevrollier A, Guillet V, Loiseau D, et al. Hereditary optic neuropathies share a common mitochondrial coupling defect. Ann Neurol. 2008;63(6):794-8.

35. •• Amati-Bonneau P, Valentino ML, Reynier P, et al.: OPA1 mutations induce mitochondrial DNA instability and optic atrophy plus phenotypes. Brain. 2008;131:338-51. This study established OPA1 as a novel mtDNA maintenance disorder. Skeletal muscle biopsies from these patients revealed high levels of cytochrome c oxidase negative fibers, secondary to the accumulation of clonally expanded $m t D N A$ deletions.

36. •• Hudson G, Amati-Bonneau P, Blakely EL, et al.: Mutation of OPA1 causes dominant optic atrophy with external ophthalmoplegia, ataxia, deafness and multiple mitochondrial DNA deletions: a novel disorder of mtDNA maintenance. Brain. 2008;131:329-37. This study established OPA1 as a novel mtDNA maintenance disorder. Skeletal muscle biopsies from these patients revealed high levels of cytochrome $c$ oxidase negative fibers, secondary to the accumulation of clonally expanded mtDNA deletions

37. •• Yu-Wai-Man P, Sitarz KS, Samuels DC, et al.: OPA1 mutations cause cytochrome $c$ oxidase deficiency due to loss of wild-type mtDNA molecules. Human Molecular Genetics. 2010;19 (15):3043-52. This study established OPA1 as a novel mtDNA maintenance disorder. Skeletal muscle biopsies from these patients revealed high levels of cytochrome c oxidase negative fibers, secondary to the accumulation of clonally expanded mtDNA deletions.

38. Chen HC, McCaffery JM, Chan DC. Mitochondrial fusion protects against neurodegeneration in the cerebellum. Cell. 2007;130 (3):548-62.

39. • Chen HC, Vermulst M, Wang YE, et al.: Mitochondrial Fusion Is Required for mtDNA Stability in Skeletal Muscle and Tolerance of mtDNA Mutations. Cell. 2010;141(2):280-9. This important study explores the fundamental roles played by mitofusins and OPA1 in both mtDNA maintenance and mitochondrial network stability. Loss of mitochondrial fusion was found to exacerbate mitochondrial dysfunction, with the induction of a compensatory mitochondrial proliferative response, and an increased rate of $m t D N A$ mutagenesis.

40. •• Elachouri G, Vidoni S, Zanna C, et al.: OPA1 links human mitochondrial genome maintenance to mtDNA replication and distribution. Genome Research. 2011;21(1):12-20. This study provides strong evidence that the peptide segment encoded by exon $4 b$ 
likely plays a crucial role in physically anchoring nucleoids to the mitochondrial inner membrane.

41. Yu-Wai-Man P, Bailie M, Atawan A, et al.: Pattern of retinal ganglion cell loss in dominant optic atrophy due to OPA1 mutations. Eye. 2011; Epub ahead of print.

42. Andrews RM, Griffiths PG, Johnson MA, Turnbull DM. Histochemical localisation of mitochondrial enzyme activity in human optic nerve and retina. Br J Ophthalmol. 1999;83(2):231-5.

43. Bristow EA, Griffiths PG, Andrews RM, et al. The distribution of mitochondrial activity in relation to optic nerve structure. Arch Ophthalmol. 2002;120(6):791-6.

44. •• Klopstock K, Yu-Wai-Man P, Dimitriadis K, et al.: A randomized placebo-controlled trial of idebenone in Leber's hereditary optic neuropathy. Brain. 2011; 134(9): 2677-86. This is the first adequately powered treatment trial for LHON, highlighting the benefit of a multinational approach and the need to develop global registries to support patient recruitment for relatively rare genetic diseases. High-dose idebenone is safe and it holds therapeutic potential for affected LHON carriers at highest risk of further visual loss.
45. Haefeli RH, Erb M, Gemperli AC, Robay D, et al. NQO1dependent redox cycling of idebenone: effects on cellular redox potential and energy levels. PLoS One. 2011;6(3): e17963.

46. Qi XP, Lewin AS, Sun L, et al. SOD2 gene transfer protects against optic neuropathy induced by deficiency of complex I. Ann Neurol. 2004;56(2):182-91.

47. Qi XP, Sun L, Hauswirth WW, et al. Use of mitochondrial antioxidant defenses for rescue of cells with a Leber hereditary optic neuropathycausing mutation. Arch Ophthalmol. 2007;125(2):268-72.

48. Ellouze S, Augustin S, Bouaita A, et al. Optimized allotopic expression of the human mitochondrial ND4 prevents blindness in a rat model of mitochondrial dysfunction. Am J Hum Genet. 2008;83(3):373-87.

49. Brown DT, Herbert M, Lamb VK, et al. Transmission of mitochondrial DNA disorders: possibilities for the future. Lancet. 2006;368(9529):87-9.

50. Schaefer AM, McFarland R, Blakely EL, et al. Prevalence of mitochondrial DNA disease in adults. Ann Neurol. 2008;63 (1):35-9. 